\title{
The expenditure of computer-related worktime using clinical decision support systems in chronic pain therapy
}

Timm Hecht, Anika C. Bundscherer, Christoph L. Lassen, Nicole Lindenberg, Bernhard M. Graf, Karl-Peter Ittner and Christoph H. R. Wiese*

\begin{abstract}
Background: Estimate the expenditure of computer-related worktime resulting from the use of clinical decision support systems (CDSS) to prevent adverse drug reactions (ADR) among patients undergoing chronic pain therapy and compare the employed check systems with respect to performance and practicability.

Methods: Data were collected retrospectively from 113 medical records of patients under chronic pain therapy during 2012/2013. Patient-specific medications were checked for potential drug-drug interactions (DDI) using two publicly available CDSS, Apotheken Umschau (AU) and Medscape (MS), and a commercially available CDSS AiDKlinik ${ }^{\circledast}$ (AID). The time needed to analyze patient pharmacotherapy for DDIs was taken with a stopwatch. Measurements included the time needed for running the analysis and printing the results. CDSS were compared with respect to the expenditure of time and usability. Only patient pharmacotherapies with at least two prescribed drugs and fitting the criteria of the corresponding CDSS were analyzed. Additionally, a qualitative evaluation of the used check systems was performed, employing a questionnaire asking five pain physicians to compare and rate the performance and practicability of the three CDSSs.
\end{abstract}

Results: The AU tool took a total of 3:55:45 $\mathrm{h}$ with an average of 0:02:32 $\mathrm{h}$ for 93 analyzed patient regimens and led to the discovery of $261 \mathrm{DDls}$. Using the Medscape interaction checker required a total of 1:28:35 $\mathrm{h}$ for 38 patients with an average of 0:01:58 $\mathrm{h}$ and a yield of 178 interactions. The CDSS AID required a total of 3:12:27 $\mathrm{h}$ for 97 patients with an average time of analysis of 0:01:59 h and the discovery of $170 \mathrm{DDls}$. According to the pain physicians the CDSS AID was chosen as the preferred tool.

Conclusions: Applying a CDSS to examine a patients drug regimen for potential DDls causes an average extra expenditure of work time of 2:09 min, which extends patient treatment time by $25 \%$ on average. Nevertheless, the authors believe that the extra expenditure of time employing a CDSS is outweighed by their benefits, including reduced ADR risks and safer clinical drug management.

Keywords: Drug-drug interaction, Clinical decision support systems (CDSS), Adverse drug reaction, Expenditure of time, Chronic pain, Polypharmacy

\section{Background}

Pharmacotherapy features prominently in patient treatment and the number of prescribed drugs has steadily increased [1]. According to literature estimates the average number is five prescribed drugs among the elderly patients $[2,3]$. However, the growing number of drugs taken by patients is

\footnotetext{
* Correspondence: christoph.wiese@ukr.de

Department of Anesthesiology, University Medical Centre Regensburg, Franz-Josef-Strauß-Allee 11, D-93053 Regensburg, Germany
}

significantly associated with the risk of drug-drug interactions (DDIs) [3-5]. These DDIs may lead to adverse drug reactions (ADR) [6-8]. For example, each year up to 20.000 people die from ADRs in Great Britain's hospitals [9], including ADRs due to DDIs. DDIs are not only an issue of polymedication but can also arise from lack of knowledge resulting in medication errors [10]. In fact, the majority of medication errors happens at the stage of prescription [11-14] making DDIs a rather common concern [12]. 
ADRs are a serious cause of mortality, morbidity and costs in the healthcare system, making them a major burden in healthcare [15-17]. Notably, there are estimates suggesting that up to $52 \%$ of the ADRs are avoidable [18]. Thus, strategies to prevent ADRs are of special interest. Clinical decision support systems (CDSS) are especially conceived for this task $[19,20]$ and could positively affect physicians' efforts concerning safe drug prescribing and the detection of DDIs [19-24]. However, not many data are available about how practicable it is to implement the usage of CDSSs in a hospital workflow. A major aspect for the decision to integrate the regular use of a CDSS in a clinical routine is the required time [11, 25-27]. Therefore, this study has compared three different CDSSs with respect to the expenditure of time, performance and practicability associated with the computer-based analysis of pharmacotherapies in order to prevent ADRs due to DDIs.

\section{Methods}

\section{Study group}

This was a retrospective clinical study in the chronic pain unit at the university hospital of Regensburg in Germany. The study population consisted of 113 patients who were undergoing chronic pain therapy at the chronic pain unit in the years $2012 / 2013$. This setting was chosen because of the special interest in ADR resulting from DDIs in multi-morbid and polymedicated patients. Patient records were viewed, extracting parameters such as age, body size, gender and weight for descriptive statistics. Furthermore, pain location and the duration of disease were recorded. Information about the patient's medication was gathered, including the type and the total amount of drugs that had been prescribed.

\section{Check systems}

For analyzing each patient's pharmacotherapy for DDIs, three CDSS were selected: the two freely available internet-based interaction check systems: (1) "Apotheken Umschau" (AU; SCHOLZ Datenbank ${ }^{\circ}$, ( $)$ ePrax AG) and (2) Medscape (MS; copyright $\odot$ by WebMD LLC), and the commercially available (3) CDSS AiDKlinik (AID; copyright (c) Dosing $\mathrm{GmbH}$ ). Other CDSS are available in Germany. However, the AU, MS and AID check systems were chosen as they are representatives of the major types of CDSS: freely or commercially available, designed for use by both patients and physicians or by heath care professionals only, applicable to drugs approved in a specific country or internationally.

(1) AU: The AU CDSS was designed for use by both patients and physicians. Therefore, the information provided is more detailed and comprehensible also for users without specific medical knowledge. The
AU CDSS was conceived for use in Germany and drugs approved in Germany only. It is a tool providing the users not only with information about DDIs of a specific pharmacotherapy under consideration but additionally about double medication, fitness to drive, drug-food interactions and cumulative side effects. Up to ten drugs can be entered and analyzed using both, active agent and trade name, dose and dosage form. This CDSS classifies occurring DDI as: (1) Severe interactions that should be avoided implicitly. These are marked by the system with a red stop-sign. (2) Severe or significant interactions, that should be avoided, marked with a red exclamation mark. (3) Interactions that are significant but only in some rare cases, marked with orange exclamation mark. (4) Interactions of notable but minor relevance, marked with a yellow exclamation mark. (5) Interactions which most probably are not existent. Additionally, information about DDI is given, comprising a summary explaining the main interaction issue. Furthermore, albeit not considered in this study, the interaction is outlined in detail, classifying the interaction, reporting about the mechanism, time-dependent course, symptoms that can appear and which dose needs to be considered. Management of the interaction is recommended explaining which afflictions and symptoms are to be looked out for, which laboratory findings are important and which actions are to be taken. The CDSS AU drug databank is based on information approved by the Federal Institute for Drugs and Medical Devices and is continuously updated albeit at irregular intervals.

(2) MS: Medscape (MS) is a freely available internetbased CDSS. It is an internationally well established tool for simple drug-drug interaction checking. It was created for English speaking countries and drugs approved in the USA only. Drugs are entered by generic name, active agent or trade name. A dose cannot be entered. The maximum number of drugs that can be analyzed is 30 . A given patient's pharmacotherapy is evaluated for DDIs. A short explanation about interaction issue and mechanism is provided. Furthermore, the tool indicates relevant aspects and risks of the patient's regimen such as deterioration of renal function or renal dysfunction, the potential for causing gastrointestinal bleedings or teratogenic effects for certain drugs given during pregnancy. The CDSS classifies the DDIs as: (1) Serious - use alternative. (2) Significant monitor closely. (3) Minor or non-significant. Of note, a DDI can be classified as serious, significant or minor at the same time when the underlying drug combinations have multiple, different effects. 
The clinical information is updated regularly and represents the expertise and practical knowledge of top physicians and pharmacists from leading academic medical centers in the United States and worldwide.

(3) AID: AID, the third evaluated CDSS, is commercially available and was designed to support healthcare professionals in a hospital workflow. Drugs are entered by their generic name, active agent, trade name and dose. More than 30 drugs can be analyzed. Besides DDIs, users are informed about double medication, incompatibility, exceeded maximal doses and the risk of kidney insufficiency due to the patient's pharmacotherapy. Furthermore, assistance is provided in drug usage during pregnancy. Interactions found are classified as (1) Clinically severe interaction, outlined red. (2) Clinically relevant interaction with potential to harm. (3) Clinically most probably not relevant interactions, outlined yellow. In addition, interactions are explained, pointing out risk patients and potential symptoms. Detailed clinical management is provided including the suggestion of alternative drugs, lowering or raising a drug dose or close monitoring if drug combinations avoided. The CDSS AID refers to the Pharmindex database which is updated every 14 days and issued by the Medizinischen Medien Informations $\mathrm{GmbH}$ (MMI, Neu-Isenburg, Germany).

\section{Pain physician questionnaire}

Additionally, the three employed CDSSs were evaluated and compared by five pain physicians at the University of Regensburg to highlight the main advantages and weaknesses of each system. Furthermore, these physicians were asked to rate main advantages of each System to determine their preferred CDSS.

\section{Time measurement}

To evaluate the expenditure of time using the CDSSs for analyzing patient's pharmacotherapy time was taken with a stopwatch. For AU and MS time measurement involved: opening the Mozilla Firefox ${ }^{\mathrm{mm}}$ (Mozilla Foundation, Mountain View, California, United States) internet browser, selecting the CDSS, entering the patient's medication, analyzing and printing the results. Printing the results in MS was eased by a print button whereas printing in AU was solely possible by text marking. Prior to their use, bookmarks for the websites of the MS and AU interaction checkers were attached to the bookmark library of the internet browser for faster access. This was neither required nor possible for AID.

For AID, the measured process contained: starting the hospital's intranet, opening the AID homepage, choosing the "medi-box" item, entering the drugs, analyzing and printing the results using the print option. All interactions found were claimed DDI whether severe or nonsignificant and irrespective of whether or not the patient had experienced an ADR. This setup was chosen to simulate the drug interaction check as practiced under a normal work routine. Only patient's regimens with at least 2 drugs and fitting the criteria of the corresponding CDSS were analyzed for DDIs.

\section{Definitions of the terms used}

Drug-Drug-Interactions (DDI) were defined as reactions occurring from drugs taken at the same time and affecting each other. These reactions can be synergistic or antagonistic and influence the intended therapeutic effect as well as increase the possibility or the extent of side effects. The major issue of DDI is the potential to harm the patient.

Adverse drug events (ADE) comprise all adverse effects caused by normal as well as improper drug use. Adverse drug reactions (ADR) are specific ADEs. ADRs were defined as negative effects caused by the combination of two or more drugs, during regular use at normal dose, with the potential to harm a patient.

Medication error was defined as every drug combination leading to a DDI with potential to harm, even if no actual harm was done, the DDI was non-significant or the probability of causing harm was very small.

\section{Statistical analysis and ethics}

Data were acquired primarily through the German Pain Questionnaire. Patient-specific parameters were encoded by a custom made system and entered in a table created with the spreadsheet program MS Excel, vs. 2010 (Microsoft Inc., Redmond, USA). For statistical analysis, data were processed by the statistics program IBM SPSS for Windows, vs. 20.0 (IBM SPSS Inc., New York, USA). To analyze statistical significance one-way ANOVA was performed. Descriptive data are presented as absolute values or percentages as indicated and partly expressed as the mean. The study was approved by the Local Ethics Commission (14-160-0069, Regensburg, Germany). According to the declaration of Helsinki, data were anonymized.

\section{Results}

To investigate the time expenditure required to check for DDIs by the use of CDSS and compare their performance three different CDSS and a cohort of 113 chronic pain patients were employed. Additionally five pain physicians evaluated each CDSS to highlight advantages and weaknesses of the different check systems. Furthermore, pain physicians evaluations were compared to determine the preferred and most practical CDSS. Because of the purpose of the study, neither the clinical 
significance, nor the relevance or the correctness of the discovered interactions were considered.

\section{Chronic pain patients}

113 records of patients undergoing pain therapy were analyzed. Descriptive statistics are shown in Table 1.

\section{Medication}

Five hundred five drugs were prescribed to 110 patients; three of the former 113 patients had no medication at all. The minimum number of drugs among the patients with medication was one prescribed drug, the maximum number was twenty. The average number of drugs per patient was 4.59. 142 different types of drugs were prescribed. Most frequently prescribed drugs were analgesics (35.4\%). The non-opioid analgesic drugs with the highest frequencies of prescriptions were Ibuprofen (31.7\%), Metamizol (19.5\%) and acetylsalicylic acid (ASS) (17.8\%). Tilidin/ Naloxone (30.9 \%) and Tramadol (27.2 \%) were the opioid-analgesics with the largest number of prescriptions. Drugs affecting the cardiovascular system (beta blockers, diuretics, statins, $\mathrm{Ca}^{2+}$-inhibitors, Angiotensin converting enzyme inhibitors (ACE-Inhibitors), Angiotensin-receptorinhibitors (AT1-inhibitors), digoxin, alpha-1-receptor inhibitors) were the second most prescribed drugs (15.6\%) followed by antidepressants (9.9\%), anticonvulsives (6.1\%), proton-pump inhibitors (6.1\%), thyroxine (2.5\%), supplements $(2.1 \%)$, benzodiazepines $(1.9 \%)$ and antidiabetics (1.9\%).

\section{CDSSs - time expenditure}

Thirteen patients had just a single prescription. Therefore, only 97 patient regimens remained for evaluation.

\section{CDSS Apotheken Umschau (AU)}

93 pharmacotherapies were checked for DDIs with the CDSS AU. Due to the limitation of ten possible entered drugs, three patient's regimens could not be evaluated. One regimen could not be evaluated because the CDSS neither recognized the drug name "Avamigran" nor its active agents. Evaluating the 93 pharmacotherapies required a total of 3:55:45 h. The maximum time for analyzing one pharmacotherapy was 6:22 min for a regimen comprising ten drugs. The minimum time was 1:02 min for a regimen comprising two drugs. The average time for analyzing one patient was $2: 32 \mathrm{~min}$.

\section{CDSS Medscape (MS)}

Because the CDSS MS did not recognize various drugs or their active agents 52 pharmacotherapies could not be analyzed. Drugs not recognized by the CDSS MS were Phenprocoumon, Biperiden, Flupirtin, Tepilta suspension, Tolperison, Glibenclamid, Melperon, Metamizol, Lipoic acid, Macrogol, Opipramol, Tetrazepam, Zopiclone, Bromazepam. Moreover, drug combinations consisting of two drugs, e.g. Ramipril/HCT that had not been recognized as a combination but had been recognized when entered individually, were analyzed each on their own. The CDSS MS required a total of 1:28:35 $\mathrm{h}$ for evaluating the remaining 45 pharmacotherapies. 8:04 min was the maximum amount of time needed to analyze a patient's regimen that covered 10 drugs. The minimum amount of time for an evaluation was 0:21 min for a regimen that comprised two drugs. The average time for analyzing one patient's regimen was 1:58 min.

Table 1 Descriptive statistics

\begin{tabular}{|c|c|c|c|c|c|c|c|}
\hline \multicolumn{2}{|l|}{ Gender $^{a}$} & \multicolumn{2}{|l|}{ Age (years) } & \multicolumn{2}{|c|}{ Mean body weight (kg) } & \multicolumn{2}{|c|}{ Patients height $(\mathrm{cm})($ mean$)$} \\
\hline male & female & (Mean) & (Range) & 80.6 & & 170.4 & \\
\hline $61(54)$ & $52(46)$ & 52.4 & 19 to 84 & & & & \\
\hline \multicolumn{8}{|c|}{ Pain complaints for ${ }^{a}$} \\
\hline$>5$ years & $2-5$ years & $1-2$ years & $1 / 2-1$ years & 1 week-1 year & & no declarati & \\
\hline $46(40.7)$ & $34(30.1)$ & $17(15)$ & $7(6.2)$ & $7(6.2)$ & & $2(1.8)$ & \\
\hline \multicolumn{8}{|c|}{ Pain localization ${ }^{a}$} \\
\hline Head & Cervical sp. & Thoracic sp. & Lumbar sp. & Upper limbs & Lower limbs & Abdominal & Whole-body \\
\hline $16(14.2)$ & $9(8)$ & $2(1.8)$ & $24(21.2)$ & $13(11.5)$ & $17(15)$ & $4(3.5)$ & $28(24.8)$ \\
\hline \multicolumn{8}{|c|}{ Maximum pain intensity ${ }^{a}$} \\
\hline $0-4$ & $5-7$ & & & $8-10$ & & no declarati & \\
\hline $3(2.7)$ & $29(25.7)$ & & & $79(69.9)$ & & $2(1.7)$ & \\
\hline \multicolumn{8}{|c|}{ Mean pain intensity ${ }^{a}$} \\
\hline $0-4$ & $5-7$ & & $8-10$ & & no declaration & Median & \\
\hline $16(14.2)$ & $47(41.6)$ & & $47(41.6)$ & & $3(2.65)$ & 7 & \\
\hline
\end{tabular}

sp spine

a Total number (percentage) 


\section{CDSS AID Klinik (AID)}

Ninety-seven patient pharmacotheapies were analyzed with the CDSS AID. All patients with two or more prescribed drugs fitted the systems criteria. All drugs were recognized and no patient regimen exceeded the limit of drugs that can be entered. Analyzing the 97 pharmacotherapies with the CDSS AID required a total of 3:12:27 h. 7.39 min was the maximum amount of time needed to evaluate one patient's regimen comprising 20 drugs. The minimum amount of time required to analyze one patient's regimen comprising a pharmacotherapy consisting of two drugs was 0:43 min. The average time needed to analyze one patient's regimen was 1:59 min.

Table 2 shows the time expenditure of all three CDSSs by comparison.

\section{CDSS related time expenditure}

Employing the CDSS AID was significantly faster than the use of CDSS AU $(p<0.05)$.

\section{Most frequently involved drug combinations and drugs CDSS AU}

When evaluating 93 pharmacotherapies comprising 446 drugs the CDSS AU discovered 261 DDIs in 63 (67.7 \%) pharmacotherapies. In $30(32.2 \%)$ patient regimens no DDI was found by the CDSS AU. The maximum number of DDIs discovered was 12 in a regimen comprising ten drugs. The minimum number was one DDI discovered in a regimen comprising two drugs. The average number of interactions was 4.14 per patient. $3.8 \%$ were classified as severe interactions, $36.3 \%$ were severe or considerable interactions, $37.9 \%$ of the discovered interactions were classified as only significant in some rare cases and $16.4 \%$ were of slight relevance. $5.3 \%$ of the discovered interactions were classified as most probably not relevant. The most frequently involved drugs were non-opioids (22\%), antidepressants (14.5\%) and opioids (10.1\%).

\section{CDSS Medscape}

The CDSS MS discovered 178 DDIs in 38 (84.4 \%) pharmacotherapies comprising 202 drugs within a range of 1 to 17 . The average amount of DDIs was 4.71 . Of the 178
DDIs $6.7 \%$ were classified as severe, $75.2 \%$ as significant and $17.9 \%$ as minor significant. Concerning all DDIs discovered by the CDSS MS, non-opioid analgesics $(44.3 \%)$ were the drugs involved most frequently, followed by beta blockers (7.8 \%) and antidepressants (7.5\%). Only in 7 (15.5\%) of the 45 evaluated patient regimen no DDI was found.

\section{CDSS AID}

In analyzing 97 pharmacotherapies comprising 492 drugs, the CDSS AID discovered 170 DDIs within a range of one to twelve DDIs in 57 (58.7 \%) pharmacotherapies. No DDI's were found in $40(41.2 \%)$ of the evaluated patient regimens. The average number of interactions was 2.98 per patient. $11.7 \%$ were clinically severe interactions, $42.9 \%$ of the DDIs were classified as potentially clinically relevant interactions, and $45.2 \%$ the discovered DDIs were clinically not relevant and not further specified. The most common drugs involved in DDIs according to the CDSS AID were non-opioids (27.9 \%), ACE-inhibitors (12.9\%) and diuretics (11.2 \%). Table 3 lists the drugs and drug combinations which most frequently contribute to DDIs.

\section{Pain physician questionnaire}

All five pain physicians believe that CDSS are important tools to prevent DDIs and improve medication safety. Four stated to use CDSSs on a regular basis. One physician stated to not use computerized check systems because of the increased workload and lack of time in hospital workflow. Two physicians have used all three check systems in the past. Two have only used CDSS AID and one physician has not used either of them.

\section{CDSS AU}

Main advantages stated by the pain physicians were that AU is freely available, is easy to handle and has s selfexplanatory layout. Disadvantages of the AU CDSS are that certain drugs are not recognized and too many irrelevant drug-drug interactions are highlighted. The explanations of potential DDIs are relatively long and written for laymen. Furthermore, no medication plans can be created and saved. Therefore, the drug regimens have to be reentered each time an interaction check is

Table 2 CDSS drug-interaction check - time expenditure

\begin{tabular}{llll}
\hline & CDSS AU & CDSS AID & CDSS Medscape \\
\hline Regimens analyzed & 93 & 97 & 45 \\
Average time per analyzed regimen & $2: 32 \mathrm{~min}$ & $1: 59 \mathrm{~min}$ & $1: 58 \mathrm{~min}$ \\
Range & Minimum: 1:02 min & Minimum: 0:43 min & Minimum: 0:21 min \\
& Maximum: 6:22 min & Maximum: 7:39 min & Maximum: 8:04 min \\
Total time & $3: 55: 45 \mathrm{~h}$ & $3: 12: 27 \mathrm{~h}$ & $1: 28: 35 \mathrm{~h}$ \\
\hline
\end{tabular}

CDSS Cinical decision suport sytem, AID CDSS AiDKlinik ${ }^{\oplus}$, AU CDSS Apotheken Umschau, MS CDSS Medscape 
Table 3 Drugs and drug combinations most frequently involved in DDIs

\begin{tabular}{|c|c|c|c|c|c|}
\hline \multicolumn{2}{|c|}{ Detecting CDSS (interactions in total) } & \multirow{2}{*}{$\begin{array}{l}\text { Drug combinations } \\
\text { ASS-Ibuprofen }\end{array}$} & \multirow{2}{*}{$\frac{\%}{80}$} & \multirow{2}{*}{$\begin{array}{l}\text { Drugs } \\
\text { ASS }\end{array}$} & \multirow{2}{*}{$\begin{array}{l}\% \\
40\end{array}$} \\
\hline $\mathrm{AU}(261)$ & Severe $(3.8 \%)$ & & & & \\
\hline & & Melperone-Ropinirole & 10 & Ibuprofen & 40 \\
\hline & & Levofloxacin-Amitriptyline & 10 & Levofloxacin & 5 \\
\hline & & & & Amitriptyline & 5 \\
\hline & & & & Ropinirole & 5 \\
\hline & & & & Melperone & 5 \\
\hline & Severe/Considerable (36.3\%) & Tramadol-Pregabalin & 3.1 & Pregablin & 8.4 \\
\hline & & Amitriptyline-Tramadol & 3.1 & Ibuprofen & 7.8 \\
\hline & & Ibuprofen-Enalapril & 3.1 & Amitriptyline & 7.3 \\
\hline & & Pregabalin-Morphine & 3.1 & & \\
\hline & Significant (rare) (37.9 \%) & Ramipril-HCT & 3 & ASS & 9.6 \\
\hline & & Pantoprazole-ASS & 3 & $\mathrm{HCT}$ & 8.5 \\
\hline & & ASS-Olmesartan & 3 & Ibuprofen & 6 \\
\hline & & Metformin-ASS & 3 & & \\
\hline & Slight (16.4 \%) & ASS-Amlodipine & 14.9 & ASS & 32.5 \\
\hline & & ASS-Ramipril & 11.6 & Amlodipine & 8.1 \\
\hline & & ASS-Bisoprolol & 9.3 & Thyroxine & 8.1 \\
\hline & & ASS-Metoprolol & 9.3 & & \\
\hline & Not relevant (5.3\%) & Pantoprazole-Diclofenac & 21.4 & Pantoprazole & 21.4 \\
\hline & & Pantoprazole-Duloxetine & 21.4 & Diclofenac & 10.7 \\
\hline & & & & Carbamazepine & 10.7 \\
\hline & & & & Duloxetine & 10.7 \\
\hline \multirow[t]{6}{*}{ MS (178) } & Severe (6.7 \%) & ASS-Ibuprofen & 50 & ASS & 25 \\
\hline & & Telmisartan-Ramipril & 16.6 & Ibuprofen & 25 \\
\hline & Significant (75.2 \%) & ASS-Ibuprofen & 8.2 & ASS & 17.5 \\
\hline & & Ibuprofen-Diclofenac & 7.5 & Ibuprofen & 16.8 \\
\hline & Minor Significant (17.9 \%) & ASS-Ibuprofen & 18.7 & Ibuprofen & 21.8 \\
\hline & & Diclofenac-Ibuprofen & 15.6 & ASS & 18.7 \\
\hline \multirow[t]{7}{*}{ AID (170) } & Severe (11.7\%) & Ibuprofen-ASS & 40 & Ibuprofen & 25 \\
\hline & & Amitriptyline-Tramadol & 15 & ASS & 20 \\
\hline & & Trimipramine-Tramadol & 10 & Tramadol & 12.5 \\
\hline & Potentially clinically relevant (42.9\%) & Ramipril-ASS & 6.8 & ASS & 11.6 \\
\hline & & HCT-Ramipril & 4.1 & Ramipril & 8.9 \\
\hline & & Enalapril-Ibuprofen & 4.1 & $\mathrm{HCT}$ & 8.2 \\
\hline & & Pregabalin-Oxycodone & 4.1 & & \\
\hline
\end{tabular}

CDSS Cinical decision suport sytem, AID CDSS AiDKlinik ${ }^{\ominus}, A U$ CDSS Apotheken Umschau, MS CDSS Medscape, HCT Hydrochlorothiazide, ASS Acetylsalicylic acid

required. Additionally, minimal errors in drug spelling are not tolerated.

\section{CDSS Medscape}

According to the pain physicians, advantages of the CDSS Medscape are its free availability, quick drug input, reasonable rating and concise explanation of interactions found. The main disadvantage was stated to be the fact that Medscape was created for English-speaking countries and drugs approved in USA only. This makes drug input for German drug names difficult and even impossible for drugs that are approved in Germany but not in the USA. Furthermore, no medication plan can be created and saved, forcing the physician to reenter all drugs every time changes are made to a patient's drug regimen and when a DDI check is required. Minor errors in drug name spelling are not tolerated. 


\section{CDSS AID}

Main advantages were stated to be the easy handling and rapid drug entry with tolerance for spelling errors of drug names. A large data base helps to select all entered drugs. Ratings of the DDIs found are reasonable and explanations of the potential interactions are concise. Additionally, links to further sources of information about the drugs entered are provided. Drug plans can be created and saved. Patients regimens are clearly represented in tables.

According to the pain physicians, the main disadvantages of the CDSS AID are that it is available only at institutions which purchased the CDSS, and that the data entry is time consuming. For example, each drug has to be entered with dose and instructions for intake.

\section{Discussion}

The immediate aim of this study was to determine the time needed to analyze pharmacotherapies of chronic pain patients for DDIs, using three different CDSSs. On a wider scale, the results of this trial serve to evaluate the usability of such tools in a daily routine.

We found that analyzing patient regimens employing the CDSS AU required on average 2:32 min detecting 4.14 interactions. The CDSS MS required 1:58 min for analyzing a pharmacotherapy detecting 4.71 DDIs in average. Using the CDSS AID to analyze pharmacotherapies required $1.59 \mathrm{~min}$ detecting 2.98 interactions on average.

The time needed to evaluate a patient's pharmacotherapy, the DDIs found, as well as the drugs involved in the DDIs all depended on the particular CDSS used. For example, data entry differs among the CDSSs. While drugs are entered with dose and trade or generic name in the CDSSs AU and AID, no dose is required in MS, which can be time saving during drug entry. However, the fact that many German trade names were not recognized by the CDSS MS had an adverse effect on time expenditure. Similarly, the fact that the user had to look up the active agent before entering a patient's regimen for drugs that were not known to the CDSS increased time expenditure and effort. Furthermore, even minor errors in the spelling of a drug name or active agent made it impossible for the CDSS MS and CDSS AU search engine to find the correct drug. This makes data entry difficult, especially when the real drug name is unknown. On the other hand, the CDSS AU automatically offers the option to enter additional information about patient diseases which requires extra time even if just declining the request. Moreover, the CDSS AU has not implemented a print option. This definitely prolonged the documentation because the desired information had to be extracted and printed using another program.
The way the results of the DDI check were provided by the three CDSSs differed as well. Both, the CDSS MS and AID delivered assorted information with more severe interactions listed first. In contrast, the CDSS AU did not sort the discovered interactions by degree of severity. This made evaluation of the results more difficult and the search for desired information more time consuming. Moreover, the number of the discovered DDIs as well as their classification varies between the check systems. The CDSS MS and AID each classify DDIs based on three degrees of severity, whereas the CDSS AU utilizes five. The number of DDIs discovered by the CDSS AU and the five categories of classification may lead to reduced awareness because too much information is provided $[28,29]$.

Concerning the usability of the CDSS MS there is an additional restriction because it was primarily designed for use in Anglo-American countries. Accordingly, in $53.6 \%$ of the evaluated patient regimens, the CDSS MS did not recognize one or more drugs of the patient's pharmacotherapies including some of the most frequently used analgesics in Germany. Even though the CDSS MS is a simple and easy to use tool for analyzing pharmacotherapies, it is of limited use for evaluating pharmacotherapies of chronic pain patients in German language area. In summary, when taking into account all different aspects, the CDSS AID turned out to be the fastest CDSS and proved superior in handling compared to the two other CDSS because it is especially tailored for a hospital workflow and to ease a physician's work. These findings match the results of the implemented questionnaire and the qualitative comparison of the three CDSS by five pain physicians. All pain physicians chose the CDSS AID as preferred interaction check system. Especially the reasonable number of interactions found and the concise descriptions of DDIs make this CDSS popular among the physicians. The features that allow to create a medication plan that can be saved and that provide additional drug information make it easy to integrate this tool and the necessary interaction checks into a physicians work routine. Both other CDSSs turned out to be less practicable for different reasons. According to the pain physicians the CDSS AU seems to be a tool more suitable for patients than physicians. Too many of the discovered potential interactions are irrelevant and explanations of potential DDIs are too long. The freely available CDSS Medscape was found to be fast and sufficient with respect to identifying relevant interactions and to providing an adequate amount of additional information. Unfortunately, many drug regimens could not be evaluated for DDIs with this tool making it impossible for physicians to exclusively rely on this check system in a daily work routine. 
The expenditure of time for analyzing patients' pharmacotherapies is of great interest especially in view of the decreasing amount of time physicians have for patient treatment. According to a German study, general practitioners spend on average $8 \mathrm{~min}$ per patient assuming a number of 45 patients per day [30]. A two minute drug interaction check would extend the average patient treatment time by $25 \%$. However, this would apply only to patients who actually receive multiple drug prescriptions. Therefore, the overall increase in temporal effort and work-load for a physician is likely to be considerably smaller. Importantly, a modest increase in treatment time must be set in relation to the large numbers of DDIs that were actually detected in the patient's pharmacotherapies and the obvious necessity to avert ADRs which in principal are preventable $[6,14,18]$. Keeping in mind that ADRs are a serious cause of morbidity, mortality and costs in healthcare [15-17] it seems more reasonable to spend a rather small amount of time for a DDI check than unnecessarily risking patient welfare or to invest a definitely greater amount of time and money in order to deal with the effects of ADRs. Indeed, just correcting a medication error in hindsight is more time consuming than an interaction check in advance [11].

\section{Limitations}

The study setup reflects the performance of CDSSs in an actual hospital workflow. However, especially with respect to the check systems AID and AU only basic functions were applied. For example, aside from the prescribed drugs, no additional information was added to specify the interaction check. This could increase time expenditure in practice. In this regard, the CDSS AID provides an advantage as it is integrated into a computerized physician order entry system. Thus, additional patient information could easily be obtained and combined with the interaction check by the system increasing the accuracy of interaction checks [20]. This could lead to time savings compared to the internet-based CDSS where additional parameters and patient information have to be entered separately by the user. It must be mentioned, though, that the DDIs found by the different CDSSs were not examined for their correctness, their relevance, or their actual ADR risk. For example, the CDSS AU identified the drug combination TramadolPregabalin as a severe risk for DDIs. Yet, the combination Tramadol-Pregabalin is part of current guidelines for pain therapy. This indicates that not every CDSSbased recommendation should be adopted without additional scrutiny which in turn can be time consuming. However, this example shows that mistakes in patient treatment can also occur from the use of CDSSs [26] and suggests that prior to implementation as treatment routine a CDSS should be carefully evaluated not only with respect to time expenditure but also for reliability.

In this study, patient regimens were entered sequentially in a single session by one person and a clear training effect was observed. This made data input significantly faster towards the final entries. However, increased time expenditure and other difficulties encountered especially during the process of getting acquainted with a new computer program could be of practical relevance. They could act as deterrents and provoke first time users to avoid the application of a CDSS. Moreover, the analysis of pharmacotherapies by the use of a CDSS was not only highly dependent on the examiner's computer skills but also on his/her prior pharmacologic knowledge. For example knowing trade names and corresponding active agents ease both extracting information from patient records and entering the regimen into the CDSS.

From a technical point of view, the use of an internetbased CDSS may lead to differences in time expenditure due to location-/user-dependent differences in connection speed. Furthermore, the CDSSs were evaluated only for the expenditure of time arising from analyzing pharmacotherapies. The CDSSs were not evaluated concerning their sensitivity and the relevance of the discovered DDIs. However, in view of the observed discrepancies among the different CDSSs concerning the detected DDIs and the groups of drugs involved, this would be of further interest in future research.

\section{Conclusion}

There are many reports that show that CDSSs undoubtedly are of relevance in preventing DDIs and their consequences. In our current study we determined the amount of time required for the use of a CDSS and compared their performance and practicability. Our results show that the commercially available CDSS AID is the preferred check system of pain physicians. Irrespectively of which CDSS is chosen, however, CDSS implementation would notably extend the time for patient treatment. In the authors' opinion, the impact of this extra expenditure of time is outweighed by the longterm patient benefits including reduced ADR risks, morbidity and mortality. Therefore the authors recommend using CDSSs on a routine basis in clinical drug management during chronic pain therapy.

\section{Competing interests}

The authors assure that there are no relations with any company, whose products are mentioned in the article, or with any company that sells a competing product. CHRW, CLL, KPI, NL declare, that they received financial support for lectures and events of the following companies: Grünenthal, Hexal, Janssen-Cilag, MSD, Mundipharma, Pfizer, Takeda, Teva. This financial support did not have any influence on the outcome of this study. 


\section{Authors' contributions}

$\mathrm{TH}, \mathrm{KPI}$, and CHRW participated in designing the study. TH and CHRW participated in collecting and entering the data. NL, CLL, AB supported in editing the manuscript. BMG co-wrote the manuscript and added important comments to the paper. All authors read and approved the final manuscript.

\section{Acknowledgements}

The authors thank A. Hecht for helpful discussions and critically reading of the manuscript.

Received: 16 December 2014 Accepted: 16 July 2015

Published online: 01 August 2015

\section{References}

1. Linjakumpu T, Hartikainen S, Klaukka T, Veijola J, Kivelä SL, Isoaho R. Use of medications and polypharmacy are increasing among the elderly. J Clin Epidemiol. 2002;55(8):809-17.

2. Boyd CM, Darer J, Boult C, Fried LP, Boult L, Wu AW. Clinical practice guidelines and quality of care for older patients with multiple comorbid diseases: implications for pay for performance. JAMA. 2005;294(6):716-24. doi:10.1001/jama.294.6.716.

3. Stewart RB, Cooper JW. Polypharmacy in the aged. Practical solutions. Drugs Aging. 1994;4(6):449-61.

4. Macedo AF, Alves C, Craveiro N, Marques FB. Multiple drug exposure as a risk factor for the seriousness of adverse drug reactions. J Nurs Manag. 2011:19(3):395-9. doi:10.1111/j.1365-2834.2011.01216.x.

5. Jose J, Rao PG. Pattern of adverse drug reactions notified by spontaneous reporting in an Indian tertiary care teaching hospital. Pharmacol Res. 2006;54(3):226-33. doi:10.1016/j.phrs.2006.05.003.

6. Obreli-Neto PR, Nobili A, de Oliveira Baldoni A, Guidoni CM, de Lyra Júnior $D P$, Pilger $D$, et al. Adverse drug reactions caused by drug-drug interactions in elderly outpatients: a prospective cohort study. Eur J Clin Pharmacol. 2012;68(12):1667-76. doi:10.1007/s00228-012-1309-3.

7. Du Souich P. In human therapy, is the drug-drug interaction or the adverse drug reaction the issue? Can J Clin Pharmacol. 2001;8(3):153-61.

8. Bucşa C, Farcaş A, Cazacu I, Leucuta D, Achimas-Cadariu A, Mogosan $C$, et al. How many potential drug-drug interactions cause adverse drug reactions in hospitalized patients? Eur. J Intern Med. 2013;24(1):27-33. doi:10.1016/j.ejim.2012.09.011.

9. Pirmohamed M, James S, Meakin S, Green C, Scott AK, Walley TJ, et al. Adverse drug reactions as cause of admission to hospital: prospective analysis of 18820 patients. BMJ. 2004;329(7456):15-9. doi:10.1136/ bmj.329.7456.15.

10. Ko Y, Malone DC, Skrepnek GH, Armstrong EP, Murphy JE, Abarca J, et al. Prescribers' knowledge of and sources of information for potential drugdrug interactions: a postal survey of US prescribers. Drug Saf. 2008;31(6):525-36.

11. Bates DW, Boyle DL, Vander Vliet MB, Schneider J, Leape L. Relationship between medication errors and adverse drug events. J Gen Intern Med. 1995;10(4):199-205.

12. von Laue NC, Schwappach DL, Koeck CM. The epidemiology of preventable adverse drug events: a review of the literature. Wien Klin Wochenschr. 2003;115(12):407-15.

13. Kanjanarat P, Winterstein AG, Johns TE, Hatton RC, Gonzalez-Rothi R, Segal R. Nature of preventable adverse drug events in hospitals: a literature review. Am J Health Syst Pharm. 2003;60(17):1750-9.

14. Lövborg H, Eriksson LR, Jönsson AK, Bradley $T$, Hägg S. A prospective analysis of the preventability of adverse drug reactions reported in Sweden. Eur J Clin Pharmacol. 2012;68(8):1183-9. doi:10.1007/s00228-012-1237-2.

15. Nazer LH, Eljaber R, Rimawi D, Hawari Fl. Adverse drug events resulting in admission to the intensive care unit in oncology patients: Incidence, characteristics and associated cost. J Oncol Pharm Pract. 2013;19(4):298-304. doi:10.1177/1078155212465995

16. Pinzón JF, Maldonado C, Díaz JA, Segura O. Costos directos e impacto sobre la morbimortalidad hospitalaria de eventos adversos prevenibles a medicamentos en una institución de tercer nivel de Bogotá (Direct costs and hospital morbimortality impact from preventable adverse drug events). Biomedica. 2011:31(3):307-15. doi:10.1590/S0120-41572011000300003.

17. Bates DW, Spell N, Cullen DJ, Burdick E, Laird N, Petersen LA, et al. The costs of adverse drug events in hospitalized patients. Adverse Drug Events Prevention Study Group. JAMA. 1997;277(4):307-11.
18. Hakkarainen KM, Hedna K, Petzold M, Hägg S. Percentage of patients with preventable adverse drug reactions and preventability of adverse drug reactions - a meta-analysis. PLoS ONE. 2012;7(3), e33236. doi:10.1371/ journal.pone.0033236.

19. van Doormaal JE, van den Bemt PM, Zaal RJ, Egberts AC, Lenderink BW, Kosterink JG, et al. The influence that electronic prescribing has on medication errors and preventable adverse drug events: an interrupted time-series study. J Am Med Inform Assoc. 2009;16(6):816-25. doi:10.1197/ jamia.M3099.

20. Fritz D, Ceschi A, Curkovic I, Huber M, Egbring M, Kullak-Ublick GA, et al. Comparative evaluation of three clinical decision support systems: prospective screening for medication errors in 100 medical inpatients. Eur J Clin Pharmacol. 2012;68(8):1209-19. doi:10.1007/s00228-012-1241-6.

21. Wright A, Feblowitz J, Phansalkar S, Liu J, Wilcox A, Keohane CA, et al. Preventability of adverse drug events involving multiple drugs using publicly available clinical decision support tools. Am J Health Syst Pharm. 2012;69(3):221-7. doi:10.2146/ajhp110084.

22. Kaushal R, Shojania KG, Bates DW. Effects of computerized physician order entry and clinical decision support systems on medication safety: a systematic review. Arch Intern Med. 2003;163(12):1409-16. doi:10.1001/ archinte.163.12.1409.

23. Classen DC, Pestotnik SL, Evans RS, Burke JP. Computerized surveillance of adverse drug events in hospital patients. JAMA. 1991;266(20):2847-51.

24. Ing Lorenzini K, Reutemann B, Samer CF, Guignard B, Bonnabry P, Dayer P, et al. Quel programme informatique de détection des interactions médicamenteuses néfastes? (Comparison of four drug interaction screening programs). Rev Med Suisse. 2012;8(358):1978-82.

25. Bates DW, Kuperman GJ, Wang S, Gandhi T, Kittler A, Volk L, et al. Ten commandments for effective clinical decision support: making the practice of evidence-based medicine a reality. J Am Med Inform Assoc. 2003;10(6):523-30. doi:10.1197/jamia.M1370.

26. Rabøl LI, Anhøj J, Pedersen A, Pedersen BL, Hellebek AH. Beslutningsstøtte til elektronisk patientmedicinering: reduceres forekomsten af medicineringsfejl? (Clinical decision support: Is the number of medication errors reduced?). Ugeskr Laeg. 2006;168(48):4179-84.

27. Lyons A, Richardson S. Clinical decision support in critical care nursing. AACN Clin Issues. 2003;14(3):295-301.

28. Kesselheim AS, Cresswell K, Phansalkar S, Bates DW, Sheikh A. Clinical decision support systems could be modified to reduce 'Alert Fatigue' while still minimizing the risk of litigation. Health Affairs. 2011;30(12):2310-7. doi:10.1377/hlthaff.2010.1111.

29. Zorina Ol, Haueis P, Greil W, Grohmann R, Kullak-Ublick GA, Russmann S. Comparative performance of two drug interaction screening programmes analysing a cross-sectional prescription dataset of 84,625 psychiatric inpatients. Drug Saf. 2013;36(4):247-58. doi:10.1007/s40264-013-0027-9.

30. Grobe T, Dörming H, Schwartz F. Barmer GEK Arztreport. http:// presse.barmer-gek.de/barmer/web/Portale/Presseportal/Subportal/ Infothek/Studien-und-Reports/Arztreport/Arztreport-2010/PDFArztreport,property=Data.pdf. 2010.

\section{Submit your next manuscript to BioMed Central and take full advantage of:}

- Convenient online submission

- Thorough peer review

- No space constraints or color figure charges

- Immediate publication on acceptance

- Inclusion in PubMed, CAS, Scopus and Google Scholar

- Research which is freely available for redistribution

Submit your manuscript at www.biomedcentral.com/submit 